\title{
Privacy Preserving Event Sequence Data Visualization using a Sankey Diagram-like Representation
}

\author{
Jia-Kai Chou* \\ University of California, Davis
}

\author{
Yang Wang ${ }^{\dagger}$ \\ University of California, Davis
}

\author{
Kwan-Liu Ma $\mathrm{Ma}^{\ddagger}$ \\ University of California, Davis
}

\begin{abstract}
Given the growing rates and richness of data being collected nowadays, it is non-trivial for data owners to determine a single best publishing granularity that presents the most value of the data while preserving its privacy. There have been extensive studies on privacy preserving algorithms in the data mining community, but relatively few have been done to provide a supervised control over the anonymization process. We present the design and evaluation of a visual interface that assists users to employ commonly used data anonymization techniques for making privacy preserving visualizations of the data. We focus on event sequence data due to its vulnerability to privacy concerns. Our visual interface is designed for data owners to examine potential privacy issues, obfuscate information as suggested by the algorithm, and fine-tune the results per their requests. Case studies using multiple datasets under different scenarios demonstrate the effectiveness of our design. These studies show that using visualization as an interface can help identify potential privacy issues, reveal underlying anonymization processes, and allow users to balance between data utility and privacy.
\end{abstract}

Keywords: Privacy Preserving Visualization, Event Sequence Data Analysis

Concepts: •Security and privacy $\rightarrow$ Social aspects of security and privacy; Privacy protections; $\bullet$ Human-centered computing $\rightarrow$ Information visualization;

\section{Introduction}

Mobile devices are widely used, in which an individual's personal information can be readily collected or recorded, such as their movement sequence. With such rich content, and the sequential/temporal nature of the data, a well-trained analyst could extract and learn about many different aspects of this individual's behaviors. Previous studies have shown that due to the high regularity of human mobility patterns [Bayir et al. 2009; Gonzalez et al. 2008; Song et al. 2010], it takes only four spatial-temporal points to uniquely identify an individual from a dataset containing half a million people [de Montjoye et al. 2013], which indicates a serious privacy leakage concern. Although there have been tremendous research efforts made in the data mining community to resolve such privacy issues, it is still not easy for the users to decide which data sanitization solution to adopt under different circumstances for

\footnotetext{
*e-mail:jkchou@ucdavis.edu

†e-mail:ywang@ucdavis.edu

‡e-mail:ma@cs.ucdavis.edu
}

Permission to make digital or hard copies of all or part of this work for personal or classroom use is granted without fee provided that copies are not made or distributed for profit or commercial advantage and that copies bear this notice and the full citation on the first page. Copyrights for components of this work owned by others than ACM must be honored. Abstracting with credit is permitted. To copy otherwise, or republish, to post on servers or to redistribute to lists, requires prior specific permission and/or a fee. Request permissions from permissions@acm.org. (c) 2016 ACM.

SA '16 Symposium on Visualization, December 05-08, 2016, Macao

ISBN: 978-1-4503-4547-7/16/12

DOI: http://dx.doi.org/10.1145/3002151.3002153 achieving a good balance between data privacy and analytical accuracy [Acs and Castelluccia 2014].

We have been motivated to study how visualization techniques could be used to support existing methods or even act as an alternative solution for privacy preservation. In the context of privacy preserving data analysis, we posit that visualization presents two main advantages over existing data mining approaches: 1) Through visualization, the data owner possesses a clearer understanding of what changes have been made to the data during the sanitization process. 2) With the assistance of visualization, it is easier to verify how much privacy and utility loss have been compromised.

Previous works have addressed the importance of taking privacy concerns into consideration when creating visualizations, and have pointed out its challenges and some future opportunities. However, the research based on creating privacy preserving visualizations for the purpose of event sequence data analysis is still lacking.

In this paper, we incorporate three commonly used data anonymization methods: k-anonymity [Sweeney 2002], ldiversity [Machanavajjhala et al. 2007], and t-closeness [Li et al. 2007], into the visualization. Potential privacy leakages in the visualization are highlighted according to the user-assigned criteria of the three data anonymization methods. We also introduce a new privacy metric that integrates the three data anonymization methods, so that data owners can intuitively adjust their desired privacy level, even without obtaining extensive knowledge regarding the privacy preservation parameters. Four operations are developed to serve the purpose of creating privacy preserving visualizations. The operations can be applied either manually to address specific privacy concerns contained in certain parts of the visualization, or automatically to resolve the privacy issues of the entire visualization based on the user-assigned privacy parameters. Finally, three case studies are conducted to demonstrate 1) the primary information conveyed by the unsanitized visualization can still be derived from our privacy preserving visualization; and 2) a better balance between data utility loss and privacy can be achieved by giving users the flexibility to decide how to the privacy issues should be addressed.

To summarize, the major contributions of our work are as follows:

1. We design a visual interface that helps users identify potential privacy concerns of the data and maintain the integrity of the data when conducting event sequence data analysis.

2. We integrate three well-known data anonymization concepts so users can intuitively configure their desired privacy level, even with limited knowledge of data anonymization theories.

3. We provide users the flexibility to change the look of the visualization either locally or globally using a variety of privacy control techniques to fit their customized needs.

\section{Related Work}

We review the literature from the perspectives of privacy preserving data publishing and privacy preserving data visualization.

\subsection{Privacy Preserving Data Publishing (PPDP)}

In the data analytics field, an abundance of research efforts [Fung et al. 2010; Gkoulalas-Divanis et al. 2014] have been dedicated to- 
wards the preservation of privacy before the publication of data. $k$ anonymity [Sweeney 2002] is one of the early models proposed for such purpose. The k-anonymity model guarantees that an individual cannot be uniquely identified from at least $k-1$ other individuals through a linking attack. The concept of $l$-diversity [Machanavajjhala et al. 2007] was later proposed to ensure that each kanonymized group obtains at least $l$ different values for the sensitive attribute. This protects the privacy of the dataset from being compromised by homogeneity attacks as well as background knowledge attacks. Li et al. [2007] presented $t$-closeness and argued that if the $l$ different sensitive values obtained by a k-anonymized group are semantically similar or have a skewed distribution over the sensitive attribute, the data's privacy is still vulnerable to attacks. Differential privacy [Dwork 2006] is a recently arising privacy-preserving mechanism. It gives a strong guarantee that the presence or absence of an individual would not significantly alter the output of a query. These privacy-preserving approaches are primarily focused on solving the privacy issues in statistical/medical databases, in which the data is stored under a relational/row-based data model.

\subsection{Privacy Preserving Data Visualization}

Recently, designing visualizations with an emphasis on data privacy preservation has begun to attract increasing attention. Dasgupta et al. [2014] suggested future opportunities and challenges on privacy preserving visualization specifically for the analysis of electronic medical records (EMRs) data. Chou and Yang [2015] and Mohanty et al. [2013] enabled the process of volume rendering to be done while keeping the content of the volume dataset to be obscured to unauthorized viewers. In information visualization literature, Dasgupta and Kosara [2011] applied the concepts of kanonymity [Sweeney 2002] and 1-diversity [Machanavajjhala et al. 2007] and proposed an adaptive visualization technique for privacy preservation in parallel coordinates. In this paper, we utilize some data sanitization concepts drawn from well-known PPDP theories. We apply those concepts and design visualization tools that facilitate the process of producing privacy preserving visualizations with event sequence data.

\section{Sankey Diagram-like Visualization for Event Sequence Data and Privacy}

We first start with the definition of event sequence data. Then, we describe the use of Sankey diagram-like visualization for analyzing event sequence data and its potential privacy issues. Next, we introduce three commonly used data anonymization models in the data mining field and explain how to incorporate them into the visualization. Finally, we present a new privacy metric which integrates the three data anonymization methods. We use a mobility dataset to generate examples for our discussion.

\subsection{Event Sequence Data}

Let $E=\left\{e_{1}, e_{2}, \ldots, e_{n}\right\}$ denote a set of events (e.g, visited regions, geospatial locations, events occured, etc.). A sequence $S=s_{1}, s_{2}, \ldots, s_{m}$ consists of a list of $m$ events that occur in sequential order while an event may appear multiple times in one sequence. To include the temporal context, we can add $T=\left\{t_{1}, t_{2}, \ldots, t_{p}\right\}$ for $p$ time points. As a result, a sequence with temporal information can be represented as a set of (event, time) pairs $S T=\left\{\left(s_{i}, t_{j}\right): s_{i} \in\right.$ $\left.E, t_{j} \in T\right\}$. Table 1(a) provides an example of a mobility sequence dataset, which describes the visited places of five students and four teachers over four time points.

With the dataset presented in Table 1(a), every individual's mobility as well as their other private information, i.e. title, can be easily derived. A simple and straightforward de-identification method is to remove the information of both the unique identifier, i.e. "ID",

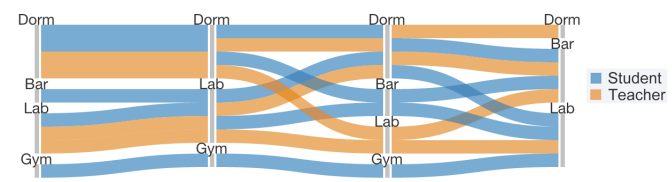

Figure 1: An example of Sankey diagram-like visualization using the data provided in Table 1.

and the temporal linkage of each individual from the dataset. As a result, the dataset is then represented by $p-1=3$ sub-tables, as shown in Table 1(b), (c), and (d), where each sub-table contains only the mobility information between any two neighboring time points $\left(t_{j}\right.$ and $\left.t_{j+1}\right)$. However, privacy issues still remain after the transformation. In the ensuing sections, we describe the visualization method that supports analysis of such type of data and discuss approaches for identifying and addressing the privacy issues contained in the dataset and the visualization.

\subsubsection{Sankey Diagram-like Visualizations}

Sankey diagram was developed for visualizing the quantitative information of flows between multiple entities or processes. When analyzing event sequence data, similar representations are adopted and focus more on presenting the subjects' quantitative change/flow between event types as well as their convergence and divergence pattern over time [Riehmann et al. 2005; Burch et al. 2013; Wongsuphasawat and Gotz 2011]. We chose to use a Sankey diagram-like representation not only because it is visually informative for presenting event sequence data but also because it inherently addresses some of the privacy issues. In the Sankey diagram-like visualization, edge crossings can negatively affect the utility of the visualization. One common strategy is to reorder the in-edges and out-edges of a node according to their source nodes and target nodes, respectively. As a result, an edge that goes into a node at the top does not guarantee that it will also leave the node at the same position, thus reducing the chance for one's mobility across multiple time points being directly traceable. Figure 1(a) shows the Sankey diagram-like visualization using the data provided in Table 1.

In addition to reordering the edges, reordering the nodes may also help reduce the visual clutter caused by edge crossings. We adopt a strategy that aims to assign the nodes that are highly-linked to each other to be neighbors. Let $n_{i}$ be a node representing a location and $N$ be the total number of nodes that represent all the unique locations in the dataset. We construct an $N \times N$ matrix, where each cell $(i, j)$ stores the total number of edges linked between $n_{i}$ and $n_{j}$ across all time points. Then, we apply algorithms for matrix reordering, such as Cuthill-McKee algorithm [1969] or hierarchical clustering [Rokach and Maimon 2005], that permute the matrix for the purpose of making cells with bigger values to be as close to the diagonal as possible. The node order in the permuted matrix is then used as the order of the nodes at each time point.

\subsection{Privacy Threats}

We consider two most commonly discussed privacy threats, identity disclosure and attribute disclosure, and use the mobility dataset shown in Table 1 to demonstrate some representative examples. In the examples, both the subjects' visited places and affilations are both regarded as potentially sensitive information. As a result, records in each sub-table that have identical values in any combination of title, $L o c_{t_{j}}$, and $L o c_{t_{j+1}}$ form an equivalence class.

\subsubsection{Identity Disclosure}

Identity disclosure means that the attacker is able to link an individual to a specific data item in the dataset. For example, in the 
(a) Original Dataset

\begin{tabular}{|c|c|c|c|c|c|}
\hline ID & Title & $L o c_{t_{0}}$ & $L o c_{t_{1}}$ & $L o c_{t_{2}}$ & $L o c_{t_{3}}$ \\
\hline 1 & S & D & D & D & B \\
\hline 2 & S & D & D & B & L \\
\hline 3 & S & L & L & B & B \\
\hline 4 & S & B & L & D & L \\
\hline 5 & S & G & G & G & L \\
\hline 6 & T & D & D & L & L \\
\hline 7 & T & D & D & D & D \\
\hline 8 & T & L & L & D & B \\
\hline 9 & T & L & L & L & B \\
\hline
\end{tabular}

(b) Mobility $t_{0}-t_{1}$

\begin{tabular}{|l|l|l|}
\hline Title & $L o c_{t_{0}}$ & Loc $_{t_{1}}$ \\
\hline S & D & D \\
\hline S & D & D \\
\hline T & D & D \\
\hline T & D & D \\
\hline S & B & L \\
\hline S & L & L \\
\hline T & L & L \\
\hline T & L & L \\
\hline S & G & G \\
\hline
\end{tabular}

(c) Mobility $t_{1}-t_{2}$

\begin{tabular}{|l|l|l|}
\hline Title & $L^{\prime} c_{t_{1}}$ & $L^{2} c_{t_{2}}$ \\
\hline S & D & D \\
\hline T & D & D \\
\hline S & D & B \\
\hline T & D & L \\
\hline S & L & D \\
\hline T & L & D \\
\hline S & L & B \\
\hline T & L & L \\
\hline S & G & G \\
\hline
\end{tabular}

(d) Mobility $t_{2}-t_{3}$

\begin{tabular}{|l|l|l|}
\hline Title & $L o c_{t_{2}}$ & $L o c_{t_{3}}$ \\
\hline T & D & D \\
\hline S & D & B \\
\hline T & D & B \\
\hline S & D & L \\
\hline S & B & B \\
\hline S & B & L \\
\hline T & L & B \\
\hline T & L & L \\
\hline S & G & L \\
\hline
\end{tabular}

Table 1: A sample mobility dataset (a) containing the location history of five students and four teachers in four time points. To anonymize the sensitive information, a straightforward de-identification method is to remove the unique identifier and the temporal linkage of each individual. As a result, sub-tables $(b),(c)$, and $(d)$, are used to represent the mobility information between any two neighboring time points.

sample dataset we can find that there is only subject that has been to "Gym" and is isolated from other subjects in most of the time points. If an attacker obtains the dataset and knows someone has been to the gym, then the attacker can find out that this subject is a student and he/she stays in the gym during the first three time points and moves to the lab at the fourth time point.

\subsubsection{Attribute Disclosure}

Attribute disclosure means that the attacker can infer an individual's sensitive information by linking the individual to a set of data items that shares some common attributes, even though not necessarily knowing the exact data item the individual belongs to. For example, from Table 1(c), we can find that at $t_{2}$ the subjects visiting the bar are all students while the subjects visiting the lab are all teachers.

\subsection{Privacy Models}

We introduce three commonly used privacy preservation models developed in the data mining field: $k$-anonymity [Sweeney 2002], $l$ diversity [Machanavajjhala et al. 2007], and t-closeness [Li et al. 2007], and show how to incorporate them into the visualization.

\subsection{1 k-anonymity}

The $k$-anonymity model [Sweeney 2002] aims at protecting the data against the identity disclosure attack. By definition, $k$-anonymity means that each equivalence class contains at least $\mathrm{k}$ records. That is, any one single record cannot be distinguished from the other $k-1$ records in the same equivalence class. To apply this analogy to mobility data, k-anonymity then means that there should always have at least $k$ people in a certain location at a certain time point. In other words, if the in-degree of a node is less than $k$, the identity of the subjects in the node may be disclosed. Figure 2(a) illustrates a simple example of identity disclosure when we set $k=2$ and we solve the issue by merging nodes. As can be seen in Figure 2(a), node $\mathrm{B}$ and node $\mathrm{C}$ both violates the 2-anonymity rule because there is less than two people entering either of them. If we merge them into one new node BC, then we can see that every node in Figure 2(a) satisfies the 2-anonymity criteria.

\subsubsection{I-diversity}

For l-diversity [Machanavajjhala et al. 2007], it is designed for protecting data against the attribute disclosure. It ensures that each equivalence class contains at least $l$ well-represented values for each sensitive attribute. In the mobility dataset example, we assume that both the location and title information of a subject can be sensitive. Therefore, we discuss them in two separate cases:

\section{Case 1 - Location Diversity}

First, we want to make sure every person's location information at any given time point is 1-diversified. That is, each node should represent at least $l$ different locations. As the example shown in Figure 2(b) where we set $l=2$, before applying the $l$-diversity criteria, one can infer that subjects in node CD must have visited node $\mathrm{A}$ at the previous time point, thus revealing the location privacy. If we merge node $A$ and node $B$ into node $A B$, such issue can then be resolved. However, the role information privacy is still leaked as one can easily tell that the subjects from node $A B$ to node $C D$ obtain the same title, and so as the people from node $\mathrm{AB}$ to node $\mathrm{EF}$. As a result, we consider the following second case.

\section{Case 2 - Title Diversity}

In this case, we want to make sure the titles in a node are $l$ diversified. Continuing with the previous example, both node $\mathrm{CD}$ and node $\mathrm{EF}$ are considered not 2-diversified as both nodes contain subjects with less than two different titles. As demonstrated in Figure 2(c), one possible solution is to merge node $\mathrm{CD}$ with node $\mathrm{EF}$ to form a new node $\mathrm{CDEF}$ in order to achieve the 2-diversity criteria.

\subsection{3 t-closeness}

t-closeness [Li et al. 2007] is also designed for protecting data against the attribute disclosure but with a different strategy. The idea is to maintain the distance between the distribution of the sensitive attribute in a class to be close enough $(<t)$ to the distribution of that attribute in the whole dataset. In the mobility dataset example, we do not take the location information into consideration because the distribution of the locations cannot be straightforwardly

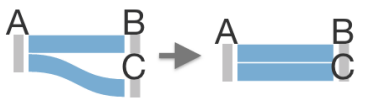

(a) $k$-anonymity $(k=2)$

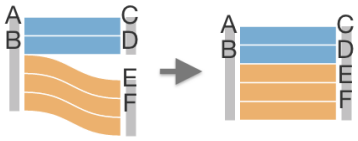

(c) l-diversity case $2(l=2)$

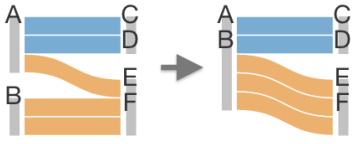

(b) l-diversity case $1(l=2)$

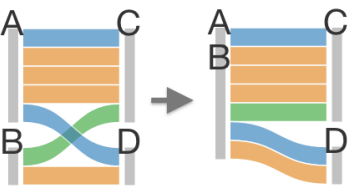

(d) $t$-closeness $(t=0.3)$
Figure 2: Examples of incorporating k-anonymity, 1-diversity and t-closeness into the sankey/storyline-like visualization. (a) Visualization satisfying 2-anonymity. (b) Visualization satisfying 2-diversity for location privacy. (c) Visualization satisfying 2diversity for title privacy. (d) Visualization satisfying 0.3-closeness. 
defined. As a result, we only calculate the distance between the distribution of the subjects' titles in a node and the corresponding distribution of those titles in the whole dataset. That is, in order to ensure $t$-closeness, for subjects who visit a certain location at a certain time point, we want to make sure that the distribution of their titles will be similar enough to the global distribution.

For example, assume that we have a dataset with a total of $n$ different titles, let $P=\left\{p_{1}, p_{2}, \ldots, p_{n}\right\}$ denote the number of subjects in each title, and $G R=\left\{g r_{1}, g r_{2}, \ldots, g r_{n}\right\}$ denote the global ratios of the titles. The global ratio of a specific title is then computed as $g r_{i}=\frac{p_{i}}{\sum p_{i}}$. For a node obtaining $m$ different titles, we count the number of subjects in each title in the node, denoted $Q=\left\{q_{1}, q_{2}, \ldots, q_{m}\right\}$. The ratios of the titles in the node, $Q R=\left\{q r_{1}, q r_{2}, \ldots, q r_{m}\right\}$ is derived similarly as the global ratios, $q r_{i}=\frac{q_{i}}{\sum q_{i}}$. Finally, we calculate the 11 -norm distance between the ratios in the node and the global ratio by only accounting for the titles appear in the node. Figure 2(d) illustrates an example when we set $t=0.3$ and assumed that the three titles, represented by orange, blue and green lines, have the ratio of $3: 2: 1=\frac{1}{2}: \frac{1}{3}: \frac{1}{6}$ in the global distribution. As a result, we can find that the distribution of the titles in node B violates the 0.3-closeness criteria. In this example, we merge node $\mathrm{B}$ with node $\mathrm{A}$ to solve this issue.

\subsection{Privacy Criteria Control}

Some may find it confusing when trying to decide the right parameters for the three data anonymization methods if not entirely familiar with the purposes of $k$-anonymity, l-diversity and $t$-closeness. According to their definitions, one should assign a bigger value to $k$ or $l$, or set a smaller $t$ value in order to reach a higher privacy requirement. The main issue is that these three parameters do not change at the same scale. For example, $t$ usually ranges from 0.0 to 1.0 while $k$ and $l$ are integers. Moreover, it is not very practical to set $k$ and $l$ to the same value as the number of records and the number of different sensitive attribute values are of different scales. To simplify the privacy control and provide another alternative, we derive a unified parameter $p$, which ranges from 0.0 to 1.0 , that integrates the three privacy criteria into one single intuitive value:

$$
\begin{aligned}
k & =\max \left(1,\left\lceil p \times k_{\max }\right\rceil\right) \\
l & =\max \left(1,\left\lceil\log _{2} k\right\rceil\right) \\
t & =\max \left(t_{\text {min }}, \frac{l_{\text {max }}}{1+(l \times p)} \times t_{\text {min }}\right)
\end{aligned}
$$

where $\lceil *\rceil$ denotes the ceilling operation, as $k_{\max }$ and $l_{\max }$ are the maximum allowed $k$ and $l$ values, respectively. $t_{\min }$ refers to the minimum allowed value of $t$. In our experiments, we set $k_{\max }=16$, $l_{\max }=4$, and $t_{\min }=0.25$. The intention of introducing $p$ is to provide users a more straightforward way to adjust their desired privacy requirement, while a smaller $p$ value means a relatively loose privacy level and a bigger $p$ value leads to a stricter privacy need.

\section{Privacy Preserving Visualization}

In this section, we discuss the strategies we adopted for generating privacy preserving visualizations. We first address the importance of maintaining the visualization utility when imposing privacy preserving operations to the visualization. Then, we introduce four privacy preserving operations that can be used in different contexts and provide users the flexibility to manipulate the visualization towards the goals of both privacy preservation and utility control.

\subsection{Visualization Utility}

Numerous metrics have been proposed to evaluate the utility and quality of data after applying anonymization/privacypreservation algorithms. In the data mining field, [Li et al. 2007] and [Machanavajjhala et al. 2007] applied the metrics of discernibility and average group size. Discernibility measures the number of records that cannot be distinguished from one another. Average group size refers to the average number of records that can be obtained from each equivalence class. Moreover, [Machanavajjhala et al. 2007] also takes both the number of generalization steps performed and the data distribution into account. From a privacy preserving visualization perspective, Dasgupta et al. [2013] stated that utility can be regarded as a function of visual uncertainty, and they introduced metrics for quantifying the visual uncertainty in clusterbased visualizations, such as scatter plot and parallel coordinates.

Although the aforementioned quantifiable numbers could be good indicators, we are inclined to utilize the major advantage offered by a visualization, i.e. to help the data owner confirm or gain a clearer understanding of the underlying anonymization process. In addition, in [Brickell and Shmatikov 2008; Li and Li 2009] the authors made similar claim that the evaluation of utility should depend on the questions that the user tries to find answers from the data, and how accurate they can be answered. We believe it is more practical and helpful for users to obtain the flexibility to determine how they wish to balance the tradeoff between privacy and utility. We argue that in different situations users may have different privacy considerations. It is important to allow them to decide how to fine-tune the visualization results so as to meet their customized requirements.

\subsection{Privacy Preserving Operations}

To help users create privacy preserving visualizations that fit their customized needs, we design four privacy preserving operations, namely Merge, Redirect, Delete, and Blur, which can be used to manipulate the visualization result to achieve the goal of privacy preservation. As increasing privacy inevitably comes at the cost of reducing certain amount of utility, the four operations are designed to handle the privacy issues in different perspectives and can be applied in different contexts. To explain, we use the sample dataset provided in Table 1 and continue from the example in Figure 3(a) where privacy-leaking nodes and edges are highlighted after the privacy criteria are set as $k=2, l=1$, and $t=0.5$.

Merge - The merge operation combines two existing nodes to form a new node. It is the most general operation among the four operations and can be applied to most cases. For example, if we look at $t_{2}$ in Figure 3(a) we can find that all the subjects in the lab are teachers, and all the subject in the bar are students. As a result, if we know someone who is in either the lab or the bar at $t_{2}$, then we know his/her title. Therefore, we decide to merge the two places to avoid such privacy leak, as the result can be seen in Figure 3(b). Depending on the context, a merge operation usually introduces uncertainty to the visualization and therefore can be used to address both identity disclosure and attribute disclosure.

Redirect - The redirect operation re-assigns a selected edge's source or target node to another user-specified node. It is especially effective when the privacy issue is only involved with one subject and that privacy issue only spans in a few or even one time point. For example, in Figure 3(b) we can see that only one subject (a teacher) stays at the dorm from $t_{2}$ to $t_{3}$. To fix this issue, we redirect the edge, which links to "dorm" at $t_{3}$, to be linked to "bar" as shown in Figure 3(c).

Delete - The delete operation eliminates the entire sequence of one individual's record. This operation works the best in the case when some subject mostly behaves differently or is isolated from the others. For example, at the bottom of Figure 3(c) we can see the person's trajectory does not intersect with any of the others. As a result, it makes the privacy of this particular person very vunerable. One can easily identify how long he/she stays in the gym and when does he/she leave the gym for the lab. We decide to delete his/her 


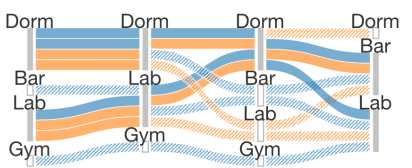

(a) Privacy highlighted Vis.

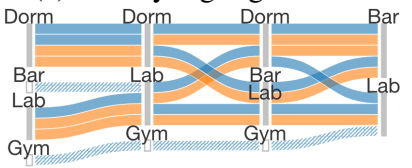

(c) Redirect an edge

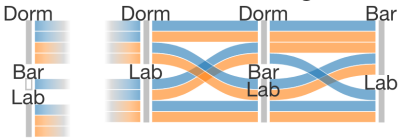

(e) Transition blurred

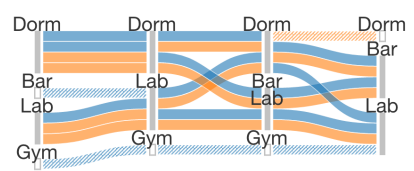

(b) Merge two nodes

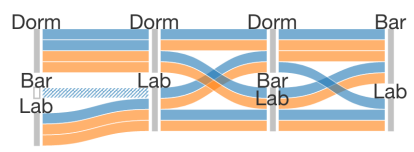

(d) Delete whole sequence

(f) Automatic approach

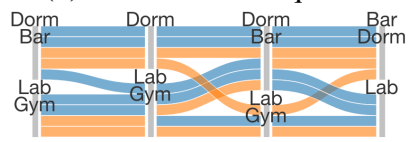

Figure 3: Examples of applying the privacy preserving operations on the sample dataset.

entire sequence as it will not change the patterns of other users. The result after deletion can be seen in Figure 3(d).

Blur - The blur operation hides the transition information between two time points but keeps the information about the number of edges in each node available. The context of using this operation is when the user does not want the viewers to know the exact flow of subjects between nodes within two time points, but still would like to preserve the pattern of the most/least visited places. In Figure 3(e), we show the example after the transition between $t_{0}$ and $t_{1}$ is blurred. We can see that the number of subjects who visited the dorm remains the same. Moreover, the distributions of the titles also does not change. However, we cannot be sure that is it because all four subjects stayed in the dorm or maybe some other subjects traveled to the dorm and some subjects left the dorm.

The application of these four privacy preserving operations are not limited to places where privacy issues occur. They can be applied to any part of the visualization. If a user feels certain part of the visualization/data requires a stronger privacy protection, the user can apply multiple operations or apply the same operation multiple times to make the privacy highly preserved in that part of the visualization.

\subsection{Automated Privacy Preservation}

We also offer the option for the users to resolve the privacy issues automatically throughout the entire visualization. We only consider the merge operation in the automatic approach as it is the most general operation and can be applied in most situations. For each node that contains some privacy issue, we search each of its neighboring nodes at the same time point as a candidate and calculate the resulting $k, l$, and $t$ values as if they were merged. Then, we decide which candidate node to be merged with the privacy leaking node first by who yields a smaller $k$ value, second by who yields a smaller $l$ value, and last by who yields a bigger $t$ value. We repeat this process until all nodes and edges satisify the user-assigned privacy criteria. The automatically generated privacy preserving result can be seen in Figure 3(f).

\section{Case Studies}

We present case studies to demonstrate how our approach can be applied to multiple scenarios while still maintaining the utility of the visualization.

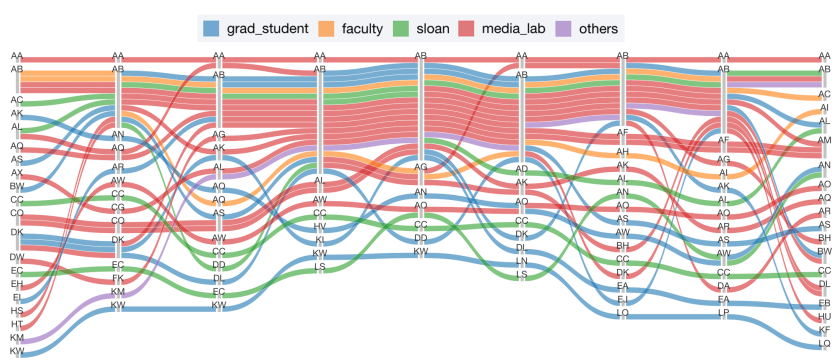

(a) Original Visualization

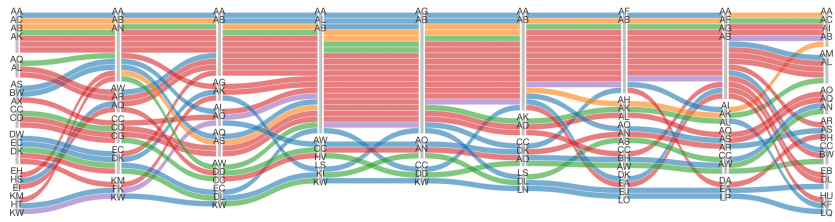

(b) Automatically Privacy Preserved Visualization

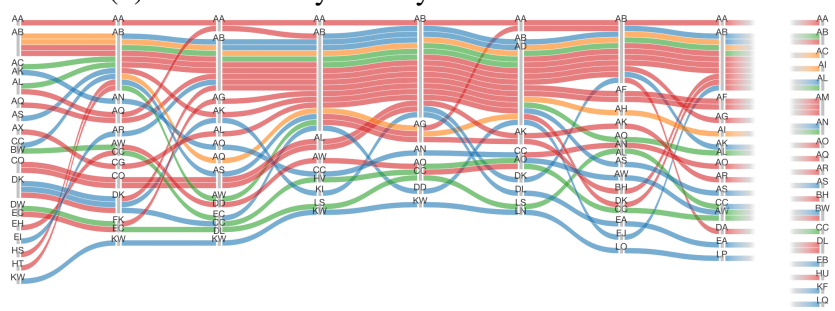

(c) Customized Privacy Preserving Visualization

Figure 4: Three visualizations showing the same data extracted from the MIT Reality mining dataset, but with different levels of privacy preservation. (a) The visualization result that presents the subjects' mobility information within a given period of time without privacy consideration. (b) The visualization result where the privacy preserving operations are applied to the entire visualization. (c) The visualization result where only specific privacy issues have been emphasized.

\subsection{MIT Reality Data}

In this case study, we use the MIT Reality Mining dataset [Eagle and (Sandy) Pentland 2006], which collects the communication, proximity, location, title and activity information from 100 subjects at MIT over the course of the 2004-2005 academic year. We pick the data of a weekday in April, 2005, which shows an interesting pattern to demonstrate our idea. We categorize the subjects' titles into five groups: "grad_student", "faculty", "sloan", "media_lab" and "others". In the dataset, the location information mostly contains only the sequence number of cell towers. We then give each cell tower a shorter name to make the identification of the nodes simpler. In addition, a subject's location at a given time point is determined by calculating the location at which the subject spent the longest amount of time during that particular hour.

Figure 4(a) shows the visualization that presents the subjects' mobility from $11 \mathrm{am}$ to $7 \mathrm{pm}$ on the selected day. We can see the pattern at a glance that during the selected time range, node $\mathrm{AB}$ is the most popular destination for many subjects, with a majority of them possessing the "media_lab" title. It would be a reasonable guess that node AB corresponds to the MIT Media Lab.

Besides the major pattern described above, other portions of the visualization show very detailed mobility routes of the users, which may lead to potential privacy leakages. In this case, we choose to apply the automated privacy preserving approach and set the $p$ value to 0.15 to obtain a visualization result with moderate privacy protection. As the privacy preserved result shown in Figure 4(b), 


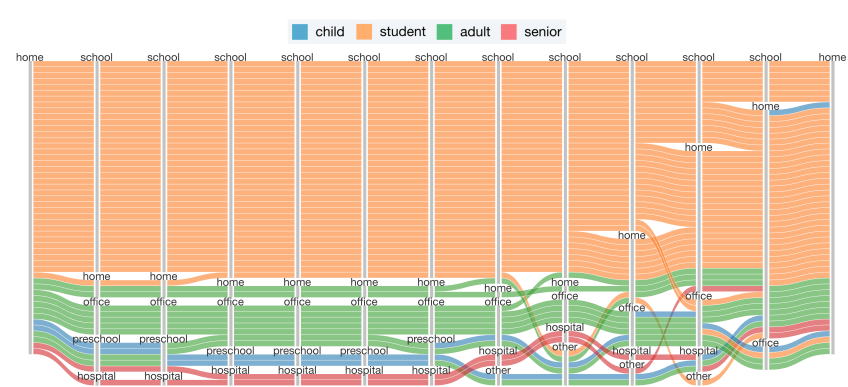

(a) Original Visualization

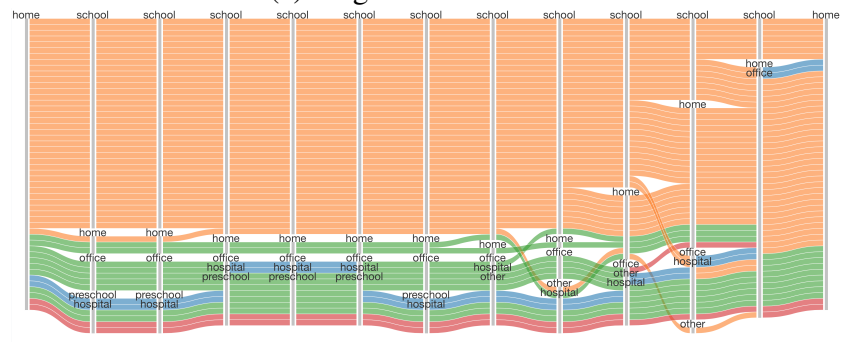

(b) Automatically Privacy Preserved Visualization

Figure 5: (a) Visualization of the daily schedule from a simulation dataset containing 50 individuals. (b) The privacy preserving visualization result after setting the $(\mathrm{k}, 1, \mathrm{t})$ values as $(1,1,0.85)$.

the major trend that many subjects travel to certain areas during some specific time periods can still be observed. However, some isolated nodes are merged and formed some smaller clusters which make the major pattern less significant. In addition, some less common places may then look more popular. This is a tradeoff between privacy and utility that the user must consider.

One might only care about certain forms of privacy enforcement. For example, assume that we only consider the location information of "sloan" students as sensitive and in need of privacy protection. As described in Section 3, we provide the flexibility for the users to make only local changes in order to meet the specific privacy preservation needs. Figure 4(c) displays a result in which the privacy of "sloan" students is emphasized. In addition, the data of "others" is deleted as there is only one subject possessing that title. Other parts of the visualization remain unchanged as in Figure 4(a).

\subsection{Simulation Data}

For this case study, we generate a synthetic population dataset that obtains 50 simulated individuals' daily schedule (7am to $7 \mathrm{pm}$ ) around a elementary school area. The 50 individuals are categorized based on their assigned demographics: "child", "student", "adult", or "senior". Their schedules are set up similar to what is done in discrete-event, agent-based simulations [Gilbert 2008]. Figure 5(a) illustrates their daily schedule as they go from their homes to school, work, or other activities, and return home for the night. From the visualization, we can easily find some interesting patterns. For example, we can find that the two children were picked up by two adults from preschool and the data indicates there are two pairs of adult and child that continue to travel together to the same locations for the rest of the day. Another example would be that one of the seniors seems to be alone at the hospital all day long. The other senior went to preschool with a kid (and maybe with another adult), then he/she went to the hospital and stayed until later afternoon. After that, he/she went somewhere to meet with the family and they headed home together.

In this type of datasets, additional care must be taken to ensure the privacy of small populations within the group. For example, chil-

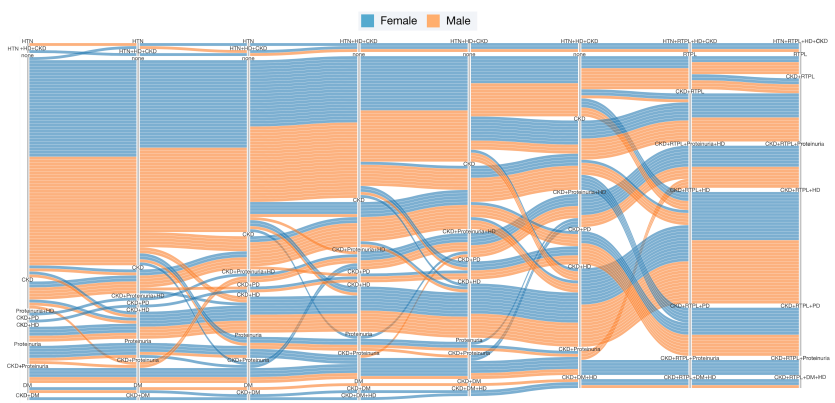

(a) Original Visualization
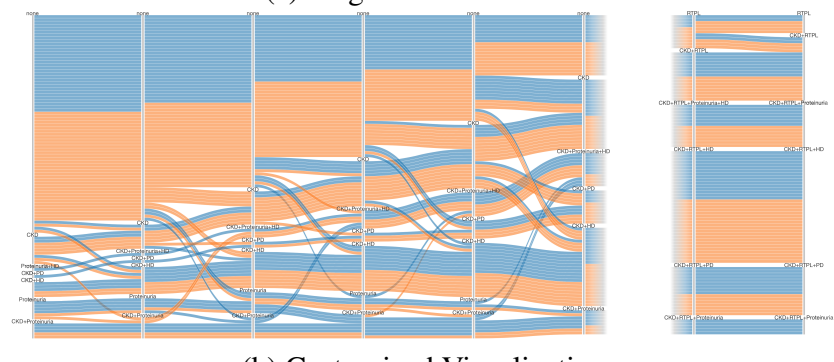

(b) Customized Visualization

Figure 6: (a) The visualization of an EMRs dataset where the patients' health histories are aligned according to the time at which a Renal Transplantation (RTPL) surgery was performed. (b) The visualization result after addressing specific privacy leaks in (a).

dren and seniors can be particularly vulnerable against privacy leakage. In addition, in this dataset there are only two members of each category within the overall population of 50 , making them easier to be identified by those would-be attackers. To solve this issue, we assign a relatively smaller $t$ value, 0.85 , while keeping the values of $k$ and $l$ at 1 . And then, we apply the privacy preserving technique to the entire visualization. Figure 5(b) shows the resulting visualization where the privacy of children and seniors are preserved as mostly other parts of the visualization remain the same.

\subsection{EMRs Data}

In this case study, we use an eletronic medical records (EMRs) dataset that contains a group of patients' medical history over a period of time. For more information about the dataset, please refer to [Wang et al. 2014]. We treat the data in a similar fashion as in [Wang et al. 2014], where diseases are represented as nodes and a patient's medical history is presented by a series of edges connecting the corresponding disease nodes at different time points. One main difference is that we apply colors to the edges rather than to the nodes in order to indicate each patient's gender. To better present our concept, a subset of the dataset was collected and the patients' disease sequences were aligned according to the time at which a Renal Transplantation (RTPL) surgery was performed. Figure 6(a) shows the visualization result without any privacy consideration as we align the time point of performing the RTPL surgery at $t_{6}$. From the visualization, we are able to discern some patterns before and after the patients underwent the $R T P L$ surgery. For example, when those patients who only suffered from Chronic Kidney Disease (CKD) underwent a RTPL surgery, some of them also performed another treatment. For those who received additional treatments, most of the female patients took Peritoneal $(P D)$ treatment while all male patients performed Hemodialysis $(H D)$ treatment.

Several privacy issues can be found in Figure 6(a). For example, by viewing the patients' flow pattern at the bottom of the visualization one will be able to deduce that if a patient is suffering from 
Diabetes Mellitus (DM), then he/she must also suffers from $C K D$, and has undergone an RTPL surgery. As a result, if an attacker obtains a prior knowledge that a particular patient is in the dataset and has $D M$, the attacker then is able to unveil the patient's confidential medical history. Another similar privacy issue can be observed at the top of the visualization. One can easily infer that if a patient is suffering from Hypertension (HTN), then he/she must also suffer from $C K D$, and has undergone RTPL and Hemodialysis (HD) surgeries. The two examples discussed above are both due to the edges being mostly isolated from the others. We decide to delete those privacy-leaking edges from the visualization, as the result after deletion can be seen in Figure 6(b).

One more potential privacy issue lies between $t_{5}$ and $t_{6}$ in Figure 6(a). If we look at the edges leaving the second from the bottom node ("CKD+Proteinuria") at $t_{5}$, we can see that for patients who suffered from $C K D$ and Proteninuria at $t_{5}$ and will perform $R T P L$ surgery at $t_{6}$, all males also receive $H D$ treatment while all females do not take any additional treatment. In this case, we choose to apply the blur operation to deal with the privacy issue mentioned above, as Figure 6(b) displays the resulting visualization. Inevitably, blurring the edges between $t_{5}$ and $t_{6}$ could lead to some utility loss as now we can only infer that the majority of the patients who underwent the RTPL surgery also suffered from $C K D$ and have performed $H D$ as well. The transition before and after the $R T P L$ sugery becomes less clear.

Two things are worth noticing when handling this type of datasets. First, because of the relative time alignment and the overlapping attributes the nodes might have, e.g. a node containing disease of "CKD" and another node containing diseases of "CKD+RTPL" are considered nodes with overlapping attributes, some privacy issues highlighted by the current $k, l, t$ model may be false positives. Therefore, further human inspection and decision-making are required. Second, in this type of datasets, the delete and blur operations may be especially suitable for protecting patients' privacy, because applying the merge or redirect operations to this type of dataset would have a higher chance of causing an undesired pattern to appear, thus potentially leading to a misdiagnosis.

\section{Discussions}

Our work thus far on privacy preserving visualization uncovers issues remaining to be addressed and suggests future research topics.

\subsection{Privacy Preserving Operations}

The four privacy preserving operations can easily be applied to other commonly used visualization techniques, such as node-link diagrams. The Sankey diagram-like visualization used in this paper can be treated as a variation of node-link diagrams where each node can have multiple edges that link to another node. The generalization of the merge, redirect, and delete operations is straightforward. For the blur operation, it would have a similar effect as applying the edge bundling on the edges between two or two groups of nodes. One future research direction would be to evaluate the strategies that how each of the operations should be carried out or how the privacy issues are identified in a node-link diagram.

Each of the four privacy preserving operations affects the utility of the visualization in a different way. The merge and blur operations both introduce uncertainty to the visualization. The merge operation adds the uncertainty to some edges' source/destination nodes while the blur operation obscures the transition of some edges. The redirect operation mostly results in information loss and sometimes may create false information gain. The delete operation removes certain information from the visualization. When applying any of the privacy preserving operations, the data owner should also con- sider the possible error/uncertainty that might cause the viewers to misinterpret the resulting visualization.

\subsection{Challenges and Future Work}

One major challenge of designing a privacy preserving visualization is how to let users interact with the visualization while still keeping the already-preserved privacy from leaking. In a Sankey diagram-like visualization, common interactive techniques include panning/scrolling, zoom in/out, filtering, and flow tracing. Panning/scrolling and zoom in/out do not affect privacy as users apply these interactions to navigate the visualization without making any change to the look of the visualization. Filtering and flow tracing, however, would introduce a counter effect with respect to privacy preservation. Both interactions are designed to allow users to only focus on the data that have certain characteristic or feature. As a result, the data that contributes to privacy preservation could possibly be filtered after the interaction. In particular, in a flow tracing interaction if we would allow the user to specifically highlight one subject's trajectory throughout the visualization, it totally contradicts the purpose of privacy preservation. We plan to address this issue in future work.

In our automatic privacy preserving approach, when a merge operation is applied to a node, we only consider its neighbor nodes as candidates and employ a greedy selection scheme to decide with whom to merge. However, in some cases merging with one of the two neighbor nodes does not always lead to a desired result. For example, in the mobility dataset if two neighboring nodes represent two physically distant locations, then merging them may not necessarily improve privacy preservation. Because it is unlikely that a subject can travel between two distant locations in a relatively short period of time, one may be able to infer how the merge operation was applied according to the context. In some other types of dataset, such as the EMRs dataset, how to sort the nodes or decide the priority of which nodes should be merged with may require domain knowledge. Otherwise, merging random neighboring nodes may result in unexpected utility loss. We believe a better algorithm can be developed by including other information of the data attributes, such as taking the hierarchical or semantic distance between data attributes into consideration.

\section{Conclusion}

We present a visual interface that incorporates the strengths of both data anonymization methods and visualization techniques. Our design helps users identify potential privacy issues, involves users in the anonymization processes, and allows users to balance their desired tradeoff between data utility and privacy. With the case studies, we demonstrate that our approach can be applied to different types of event sequence datasets. We are able to produce visualizations with different levels of privacy protection while still maintaining reasonable visual analytics ability as compared to the unsanitized visualizations. As personal data has become easily and largely collected nowadays, providing such a tool or interface like ours is important for data owners to be able to exercise control over privacy and the release of their information.

\section{Acknowledgements}

This research is supported in part by the U.S. National Science Foundation via grants NSF IIS-1528203 and NSF IIS-1320229, and the U.S. Department of Energy through grants DE-FC0212ER26072 and DE-SC0012610. 


\section{References}

Acs, G., And Castelluccia, C. 2014. A Case Study: Privacy Preserving Release of Spatio-temporal Density in Paris. In Proceedings of ACM SIGKDD '14, 1679-1688.

Bayir, M. A., Demirbas, M., and Eagle, N. 2009. Discovering spatiotemporal mobility profiles of cellphone users. In WOWMOM, 1-9.

BRickell, J., AND Shmatikov, V. 2008. The cost of privacy: Destruction of data-mining utility in anonymized data publishing. In Proceedings of ACM SIGKDD 08, 70-78.

Burch, M., Kull, A., And Weiskopf, D. 2013. Aoi rivers for visualizing dynamic eye gaze frequencies. In Proceedings of Eurographics '13, 281-290.

ChOU, J.-K., AND YANG, C.-K. 2015. Obfuscated volume rendering. The Visual Computer (Online First), 1-12.

Cuthill, E., AND McKeE, J. 1969. Reducing the bandwidth of sparse symmetric matrices. In Proceedings of the 24th National Conference, 157-172.

Dasgupta, A., And Kosara, R. 2011. Adaptive privacypreserving visualization using parallel coordinates. IEEE Trans. Vis. Comput. Graph. 17, 12, 2241-2248.

Dasgupta, A., Chen, M., And Kosara, R. 2013. Measuring privacy and utility in privacy-preserving visualization. Comput. Graph. Forum 32, 8, 35-47.

Dasgupta, A., Maguire, E., Abdul-Rahman, A., And CHEN, M. 2014. Opportunities and challenges for privacypreserving visualization of electronic health record data. In IEEE VIS 2014 Workshop on Visualization of Electronic Health Records.

De Montjoye, Y.-A., Hidalgo, C. A., Verleysen, M., And BLONDEL, V. D. 2013. Unique in the crowd: The privacy bounds of human mobility. Sci. Rep. 3 (03).

DwORK, C. 2006. Differential privacy. In 33rd International Colloquium on Automata, Languages and Programming, part II (ICALP 2006), vol. 4052 of Lecture Notes in Computer Science, $1-12$.

Eagle, N., And (Sandy) Pentland, A. 2006. Reality mining: Sensing complex social systems. Personal Ubiquitous Comput. 10, 4 (Mar.), 255-268.

Fung, B. C. M., Wang, K., Chen, R., And Yu, P. S. 2010. Privacy-preserving data publishing: A survey of recent developments. ACM Comput. Surv. 42, 4 (June), 14:1-14:53.

Gilbert, G. N. 2008. Agent-based models. Quantitative applications in the social sciences. Sage Publishing.

Gkoulalas-Divanis, A., Loukides, G., And Sun, J. 2014. Publishing data from electronic health records while preserving privacy: A survey of algorithms. Journal of Biomedical Informatics 50, 0, 4-19. Special Issue on Informatics Methods in Medical Privacy.

Gonzalez, M. C., Hidalgo, C. A., And Barabasi, A.-L. 2008. Understanding individual human mobility patterns. Nature 453, 7196 (06), 779-782.

LI, T., AND LI, N. 2009. On the tradeoff between privacy and utility in data publishing. In Proceedings of ACM SIGKDD '09, 517-526.
Li, N., Li, T., and Venkatasubramanian, S. 2007. tcloseness: Privacy beyond k-anonymity and l-diversity. In Proceedings of ICDE 07, 106-115.

Machanavajuhala, A., Kifer, D., Gehrke, J., And VenkiTASubramaniam, M. 2007. L-diversity: Privacy beyond kanonymity. ACM Trans. Knowl. Discov. Data 1, 1 (mar).

Mohanty, M., Ooi, W. T., And Atrey, P. 2013. Secure cloudbased volume ray-casting. In Proceedings of IEEE CloudCom '13, 531-538.

RiehmanN, P., Hanfler, M., AND Froehlich, B. 2005. Interactive sankey diagrams. In Proceedings of InfoVis '05, 233-240.

RoKACH, L., AND Maimon, O. 2005. Data Mining and Knowledge Discovery Handbook. Springer US, 321-352.

Song, C., Qu, Z., Blumm, N., ANd Barabsi, A.-L. 2010. Limits of predictability in human mobility. Science 327, 5968, 1018-1021.

SWEENEY, L. 2002. K-anonymity: A model for protecting privacy. Int. J. Uncertain. Fuzziness Knowl.-Based Syst. 10, 5 (Oct.), 557-570.

WAnG, C.-F., LI, J., MA, K.-L., HuAnG, C.-W., AND LI, Y.C. 2014. A visual analysis approach to cohort study of electronic patient records. In 2014 IEEE International Conference on Bioinformatics and Biomedicine (BIBM), 521-528.

Wongsuphasawat, K., AND Gotz, D. 2011. Outflow: Visualizing patient flow by symptoms and outcome. IEEE VisWeek Workshop on Visual Analytics in Healthcare, 25-28. 